\title{
Revisiting the escape speed impact on dark matter direct detection
}

\author{
Stefano Magni* \\ Laboratoir Univers et Particules de Montpellier, UMR-5299, Montpellier, France \\ E-mail: stefano.magni@univ-montp2.fr

\section{Julien Lavalle} \\ Laboratoir Univers et Particules de Montpellier, UMR-5299, Montpellier, France \\ E-mail: lavalle@in2p3.fr
}

\begin{abstract}
The knowledge of the high velocity tail of the WIMP (Weakly Interacting Massive Particles) velocity distribution has a strong impact on the way dark matter direct detection (DMDD) may constrain or discover light WIMPs in the GeV mass range. Recently, there have been important observational efforts to estimate the Galactic escape speed at the position of the Earth, like for instance the analysis published in early 2014 by the RAVE Collaboration (Piffl et al., 2014), which is of interest in the perspective of reducing the astrophysical uncertainties in DMDD. Nevertheless, these new estimates cannot be used blindly as they rely on assumptions on the Milky Way mass distribution, which induce tight correlations between the escape speed and other local astrophysical parameters (circular speed and dark matter density). We make a self-consistent study of the implications of the RAVE results on DMDD assuming isotropic DM velocity distributions, both Maxwellian and ergodic. Taking as reference the experimental sensitivities currently achieved by LUX, CRESST2, and SuperCDMS, we show that the uncertainties inferred for the exclusion curves in the low WIMP mass region are moderate, ranging from $\sim \pm 10 \%$ to $\sim \pm 20 \%$, and that the RAVE results imply large values of $\rho_{\odot}$, and so correspond to exclusion curves that are more constraining than the standard ones by $\sim 40 \%$.
\end{abstract}

Frontiers of Fundamental Physics 14

15-18 July 2014

Aix Marseille University (AMU) Saint-Charles Campus, Marseille, France

${ }^{*}$ Speaker. 


\section{Introduction}

DMDD aims at detecting WIMPs via their scattering off nuclei. A careful investigation of the physics affecting the low WIMP mass region of the parameter space for the spin-independent interpretation of this scattering is fundamental, because above $\sim 10 \mathrm{GeV}$ signal-like events reported by some experiments, in particular DAMA [1], are in contrast with other limits.

Different effects impact DMDD limits at low WIMP masses. Those related to the detector are the energy threshold and the energy resolution of the detector, while those of astrophysical origin are related to the high velocity tail of the DM speed distribution and to the local DM density. Two quantities are particularly relevant: the local escape speed from the Milky Way (MW), i.e. the speed above which particles may escape the gravitational potential of the MW, and the local circular speed, as the sum of both defines the maximum speed in the observer's frame. While the latter has been studied in depth by many authors, that is not the case for the former. A method to measure it is to use nearby high-velocity stars, that are supposed to trace the high velocity tail of the stars speed distribution, which should vanish at the escape speed as well.

Following this approach, the RAVE collaboration has published in 2014 the latest estimate of this quantity [2] (P14 from now on). Directly using those results to compute DMDD limits would be straightforward, but it would lead to inconsistent results because it would neglect the hypotheses these estimates rely upon. In our work [3] we have analyzed these assumptions and derived a selfconsistent model for the local phase-space of the DM, which consistently takes into account the correlations between the astrophysical parameters. We have computed the corresponding exclusion curves, with associated uncertainties, for the three most constraining experiments at present.

\section{Milky Way Mass Model from Rave analysis}

P14 analysis is based on a sample of $\sim 100$ stars mostly taken from the RAVE catalog. The escape speed for a star at position $\vec{r}$ can be defined as $v_{\text {esc }}(\vec{r}) \doteq \sqrt{2|\Phi(\vec{r})|}$, where $\Phi(\vec{r})$ is the gravitational potential of the MW. To derive observational constraints on $v_{\mathrm{esc}}$ from stellar velocities it is necessary to make an assumption for the shape of the high velocity tail of the stars speed distribution, and in their likelihood analysis P14 used the ansatz $f_{\star}(v) \propto\left(v_{\mathrm{esc}}-v\right)^{k}$, with $k$ calibrated from cosmological simulations.

To estimate the escape speed at the position of the Sun, P14 had to rescale the escape speed of the observed stars using the gravitational potential of the MW, for which a particular model had to be assumed. They thus transformed the line of sight velocity $v_{\|}(\vec{r})$ of each star according to $v_{\|}^{\prime}(\vec{r})=v_{\|}(\vec{r}) \times \sqrt{\left|\Phi\left(\vec{r}_{\odot}\right) / \Phi(\vec{r})\right|}$, where $\vec{r}_{\odot}$ is the position of the Sun. This correction introduces a dependence on the Milky Way mass model (MWM) assumed, which introduces specific correlations in the astrophysical parameters relevant to DMDD that one must therefore take into account when using P14 results.

P14 fixed the Sun's distance from the Galactic center $r_{\odot}=8.28 \mathrm{kpc}$ [9], the peculiar motion of the Sun [10], and they repeated the likelihood analysis considering three cases: 1) $v_{\mathrm{c}}=220 \mathrm{~km} / \mathrm{s}$, 2) $v_{\mathrm{c}}=240 \mathrm{~km} / \mathrm{s}$ and finally 3 ) free $v_{\mathrm{c}}$.

The MWM assumed in P14 contains a NFW halo for the dark matter, an Hernquist profile [7] for the baryonic bulge and halo, and a Miyamoto Nagai profile [8] for the baryonic disk. The scale density $\rho_{s}$ and the scale radius $r_{s}$ of the NFW profile are left free to vary. On the other hand, the 
baryonic content is fixed, with $M_{H}=1.5 \times 10^{10} M_{\odot}$ and $M_{\mathrm{MN}}=5 \times 10^{10} M_{\odot}$ for the total masses and $a_{H}=0.6 \mathrm{kpc}, a_{\mathrm{MN}}=4 \mathrm{kpc}$ and $b_{\mathrm{MN}}=0.3 \mathrm{kpc}$ for the scale parameters of the Hernquist bulge and of the Miyamoto Nagai disk. The MWM assumed has thus only two free parameters.

The speed of a body which is on a circular orbit on the Galactic plane can be calculated from the Newtonian gravitational potential of the MW using: $v_{\mathrm{c}}^{2}(R, 0)=\left.R \frac{d \Phi(R, z)}{d R}\right|_{z=0}$, where $R$ and $z$ are cylindrical coordinates $\left(r=\sqrt{R^{2}+z^{2}}\right)$. The escape speed is set by the kinetic energy an object needs to get unbound, i.e. to reach a certain $R_{\max }$; it is thus defined as: $v_{\text {esc }}\left(r_{\odot}\right) \doteq$ $\sqrt{2\left|\Phi\left(r_{\odot}\right)-\Phi\left(R_{\max }\right)\right|}$. To take into account the presence of nearby galaxies, the above distance is chosen to be $R_{\max }=3 R_{340}$.

Since the assumed MWM has only two free parameters, the above equations allow to convert a pair of $\rho_{s}, r_{s}$ (or equivalently a pair of $M_{340}, c_{340}$ ) into a pair of $v_{\mathrm{c}}, v_{\mathrm{esc}}$. The results of the P14 analysis with free $v_{\mathrm{c}}$, shown in Fig.13 of [2], can thus be directly converted in that plane, shown in Fig.1. It is clear from this figure that, because of the assumed MWM, the results of P14 induces strong correlations among $v_{\mathrm{c}}, v_{\mathrm{esc}}$ and the local dark matter density $\rho_{\odot}=\rho\left(r_{\odot}\right)$.

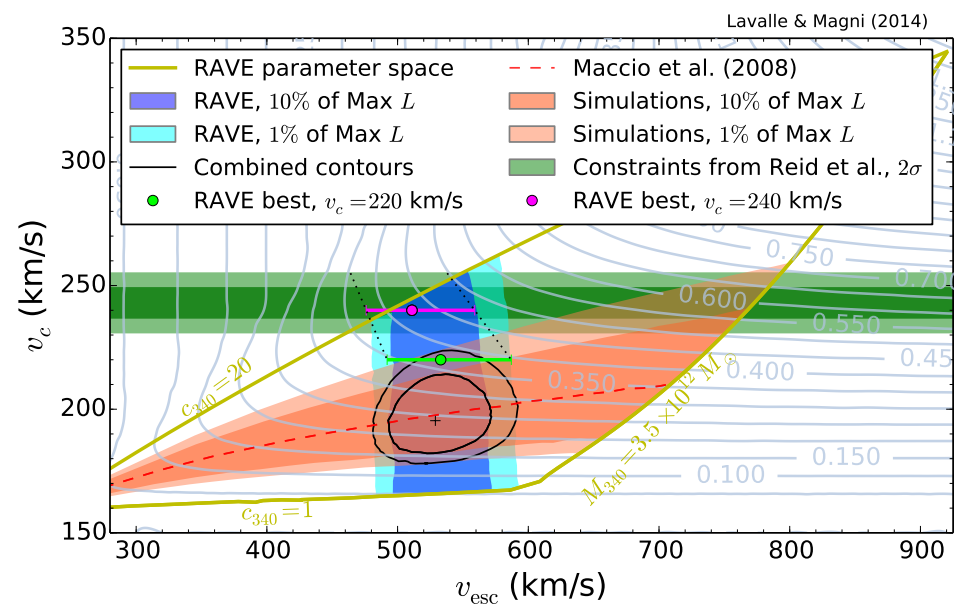

Figure 1: P14 parameter space (yellow contours), with the regions where their likelihood for free $v_{\mathrm{c}}$ decreases down to the $10 \%$ (blue) and $1 \%$ (cyan) of its maximum, and best-fit P14 results for fixed $v_{\mathrm{c}}=220$ $\mathrm{km} / \mathrm{s}$ and $v_{\mathrm{c}}=240 \mathrm{~km} / \mathrm{s}$ (with $90 \%$ C.L. error bars). Curves of constant $\rho_{\odot}$ (in $\mathrm{GeV} / \mathrm{cm}^{3}$ ) are in gray.

\section{DMDD limits from P14 results and related astrophysical uncertainties}

We now want to translate the P14 estimates into DMDD limits, focusing on the spin-independent interpretation of the elastic scattering of a WIMP of mass $m_{\chi}$ off a nucleus of atomic number $A$ and mass $m_{A}$, and no isospin violation. Thus we need to compute the differential event rate per atomic target mass in an experiment:

$$
\frac{d R}{d E_{r}}\left(E_{r}\right)=\frac{\rho_{\odot} \sigma_{p}}{2 m_{\chi} \mu_{\mathrm{p}}^{2}} F^{2}\left(E_{r}\right) \int_{|\vec{v}|>v_{\min }\left(E_{r}\right)} d^{3} \vec{v} \frac{f_{\oplus}(\vec{v}, t)}{v},
$$

where $\mu_{\mathrm{p}}$ is the WIMP-proton reduced mass, $E_{r}$ the recoil energy, $\sigma_{p}$ the WIMP-nucleon cross section, $F\left(E_{r}\right)$ the nuclear form factor (assumed of the Helm type), and $v_{\min }=\sqrt{m_{A} E_{r} /\left(2 \mu_{\mathrm{p}}\right)}$ the minimal velocity that a WIMP of mass $m_{\chi}$ needs to transfer to a nucleus of mass $m_{A}$ the recoil 
energy $E_{r} . f_{\oplus}(\vec{v}, t)$ is the dark matter velocity distribution in the Earth reference frame, obtained from the one in the Galactic frame via a Galilean transformation. In addition, one takes into account the experimental efficiency, the energy resolution of the detector, the fractions of atomic targets, the isotopic compositions for each target element, and we take the average of Eq. 3.1.

Usually, DMDD limits are computed by means of the so-called Standard Halo Model (SHM), a set of standard assumptions in which the WIMP velocity distribution is assumed to be a truncated Maxwell-Boltzmann (MB), which is based on the assumption of an isothermal sphere in the Galactic frame. It reads:

$$
f(\vec{v}) \doteq \frac{1}{N_{\mathrm{esc}}}\left(\frac{1}{\pi v_{\mathrm{c}}^{2}}\right)^{3 / 2}\left[e^{-|\vec{v}|^{2} / v_{\mathrm{c}}^{2}}-e^{-v_{\mathrm{esc}}^{2} / v_{\mathrm{c}}^{2}}\right] \Theta\left(v_{\mathrm{esc}}-|\vec{v}|\right),
$$

where $N_{\text {esc }}$ allows the normalization to unity and $\Theta$ is the Heavyside step function. In addition to the velocity distribution, the SHM fixes the values of the following parameters: $\rho_{\odot}^{S H M}=0.3 \mathrm{GeV} / \mathrm{cm}^{3}$, $v_{\mathrm{c}}^{S H M}=220 \mathrm{~km} / \mathrm{s}$ and $v_{\mathrm{esc}}^{S H M}=544 \mathrm{~km} / \mathrm{s}$. Because of the by-hand cutoff at the escape speed, the MB distribution is no more a solution of the Jeans equation, so it is not even self-consistent.

In order to build a self-consistent velocity distribution, we are going to consider functions of integrals of motion, which automatically satisfy the Jeans equation. Assuming spherical symmetry and velocity isotropy, the phase-space distribution becomes a function of the total energy $E=m \Phi+\frac{1}{2} m v^{2}$ only, which is an integral of motion. Such systems are called ergodic. Under these assumptions we can use Eddington equation, which allows to compute the phase-space distribution for the dark matter directly from the assumed gravitational potential of the Milky Way $\Phi$ and mass density profile of the dark matter $\rho$. This equation reads:

$$
f(\varepsilon)=\frac{1}{\sqrt{8} \pi^{2}}\left[\int_{0}^{\varepsilon} \frac{d^{2} \rho}{d \Psi^{2}} \frac{d \Psi}{\sqrt{\varepsilon-\Psi}}+\frac{1}{\sqrt{\varepsilon}}\left(\frac{d \rho}{d \Psi}\right)_{\Psi=0}\right] .
$$

where $\Psi=-\Phi+\Phi_{0}$ is the relative gravitational potential of the $\mathrm{MW}, \varepsilon=-E / m+\Phi_{0}$ is the relative energy per unit mass, and $\Phi_{0}$ is a constant. We can now compute the local velocity distribution for the DM as $f_{\text {erg }}\left(v, r_{\odot}\right)=f(\varepsilon) / \rho(r)$. This procedure can be applied only to spherically symmetric systems. Our MWM is not spherically symmetric, because of the disk, but since this does not dominate it can be shown that we can force spherical symmetry while not affecting the circular velocity at the Sun position.

\section{Results and discussion}

We have converted P14 results and self-consistently used them to derive DMDD exclusion curves, focusing on LUX [4] (Xe), SuperCDMS [5] (Ge) and CRESST II [6] (multi-target). The changes with respect to the SHM are both in the WIMP velocity distribution and $\rho_{\odot}$. How different astrophysical parameters affect the exclusion curves can be understood from Eq. 3.1. The sum $v_{\mathrm{esc}}+v_{\mathrm{c}}$ impacts on the position of the asymptote $\sigma_{\max } \rightarrow \infty$ on the WIMP mass axis. $v_{\mathrm{c}}$ impacts on the position of the maximum of sensitivity of the experiment on the same axis. The larger the velocity dispersion, the larger the sensitivity peak. Finally, the local dark matter density $\rho_{\odot}$ produces a global linear vertical translation of the entire curve.

Let us consider the best-fit point with prior $v_{\mathrm{c}}=240 \mathrm{~km} / \mathrm{s}$, which is likely the most motivated given the recent estimates [11]. Fig.2 shows the exclusion curves with associated 90\% C.L. uncertainties for this configuration. Comparing our results with those obtained for the SHM, we find 
out that the former are more constraining by $\sim 40 \%$ in a wide range of high WIMP masses, due to the value of $\rho_{\odot}=0.43 \pm 0.05 \mathrm{GeV} / \mathrm{cm}^{3}$, higher than the SHM one. Conversely, at low WIMP masses the SHM beats the ergodic one due to the higher $v_{\text {esc }}+v_{\mathrm{c}}$. We also show the effect of using a MB velocity distribution (instead of a more consistent ergodic one) together with the correctly correlated astrophysical parameters. We see that there is an impact especially at low WIMP masses, because of significant differences in the high-velocity tail between both distributions. The uncertainties saturate at $\sim \pm 10 \%$ at high WIMP masses, value set by the allowed range in $\rho_{\odot}$, and they degrade toward very low WIMP masses, where the maximum possible recoil energy approaches the treshold energy. It is worth noticing that some of the bumps in Fig.2 in the case of CRESST2 come from the presence of more than one target nucleus (while the others come from the energy distribution of the few nuclear recoil events collected in the experiment). This shows that employing different target nuclei in a detector helps to reduce the astrophysical uncertainties. A similar complementarity arises when considering experiments based on different target nuclei.

We now consider the $v_{\mathrm{c}}$ free analysis of $\mathrm{P} 14$, which will provide the more conservative results once translated into DMDD limits. We do not use the same prior on the concentration of the DM halo of P14 (pink band in Fig.1) because it is based on cosmological simulations without baryons. We consider the entire region provided by the $v_{\mathrm{c}}$ free analysis of RAVE in the plane of Fig.1, and combine it with the constraint on $v_{\mathrm{c}}$ published in [11], which is independent on any MWM because based on geometric quantities, namely parallaxes and proper motions of masers. The range obtained in that work is $v_{\mathrm{c}}=243 \pm 12 \mathrm{~km} / \mathrm{s}$ at $2 \sigma$, reported as a green band in Fig.1. To ensure consistency, we have verified that the other quantities estimated in [11], in particular the Sun distance from the Galactic center and the local radial derivative of the circular velocity, have values that are compatible with those of our MWM. To compute the DMDD limits associated to the $v_{\mathrm{c}}$ free analysis of P14, we use the region of the plane in Fig.1 where the RAVE blue band crosses the green band associated with [11]. There high values of the local dark matter density are allowed, up to $\rho_{\odot}=0.55 \mathrm{GeV} / \mathrm{cm}^{3}$, which are higher than those of the SHM, but in agreement with those found in recent studies [12], and which improve the potential of DMDD experiments. These results, translated into DMDD limits, are shown in Fig.2 (bottom line). The behavior is qualitatively similar to the one already described for the $v_{\mathrm{c}}=240 \mathrm{~km} / \mathrm{s}$ case, but now the uncertainties saturates at values of $\pm 20 \%$, due to the allowed range of $\rho_{\odot} \in[0.37,0.57] \mathrm{GeV} / \mathrm{cm}^{3}$.

\section{Conclusions}

We have presented a method to use the local escape speed estimates of P14 in deriving DMDD limits. A naive use of these estimates would neglect the underlying assumptions, and thus the correlations among the astrophysical parameters and the DM velocity distribution that they induce. We have found that a consistent use of these estimates implies large values for $\rho_{\odot}$, and so more constraining exclusion curves, and evaluated the associated uncertainties. The main limitations of our analysis are the simple MWM, its fixed baryonic content and the assumption that the DM phasespace be entirely governed by the total energy. We are now generalising this work to anisotropic velocity distributions, and testing the impact of the MWM using cosmological simulations. 

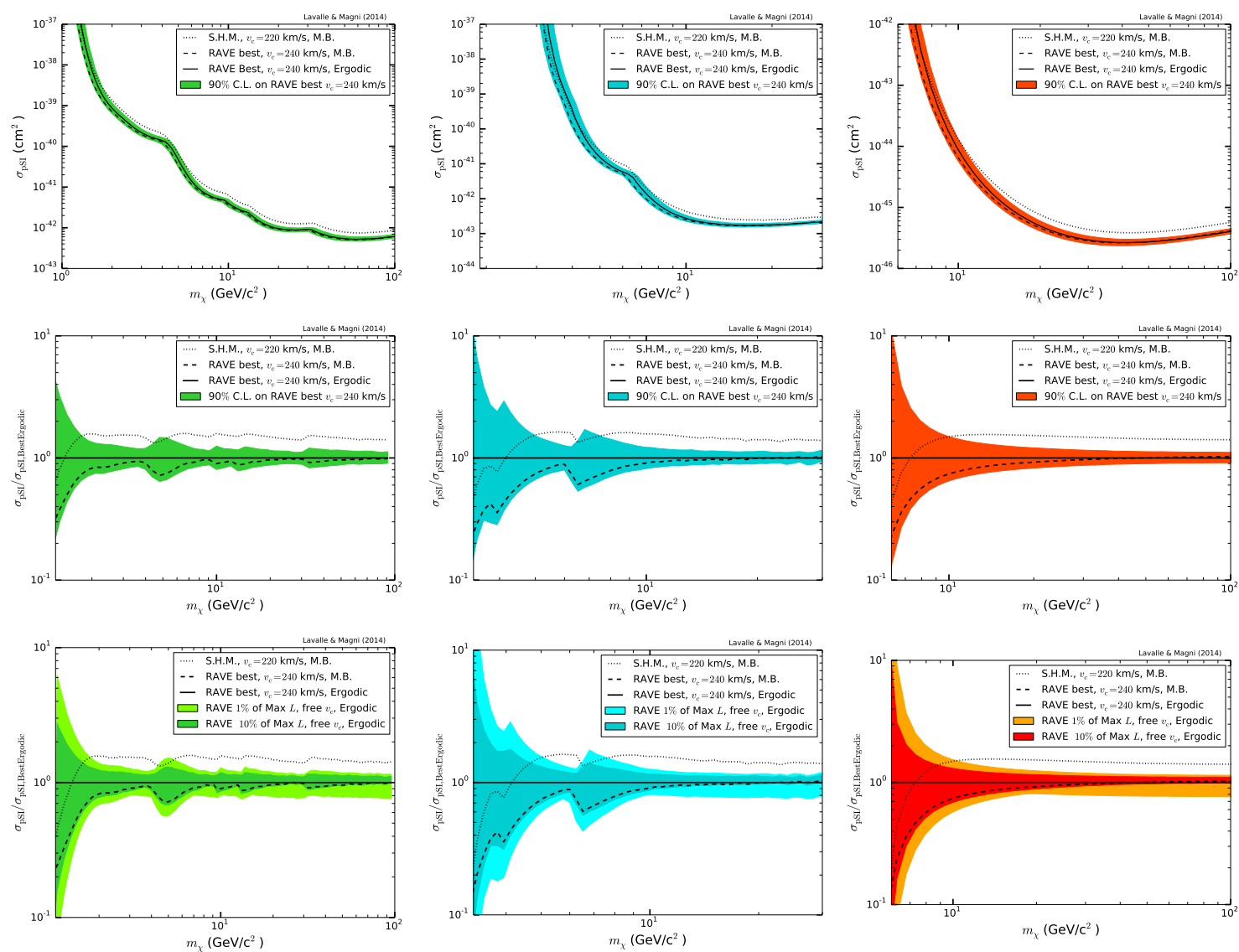

Figure 2: Experimental 90\% C.L. exclusion curves, calculated using the P14 result for the $v_{\mathrm{c}}=240 \mathrm{~km} / \mathrm{s}$ analysis (upper: absolute, middle: relative) or with a combination of the P14 $v_{\mathrm{c}}$ free analysis with additional constraints on $v_{\mathrm{c}}$ (lower: relative). Left: CRESST2, center: SuperCDMS, right: LUX.

\section{References}

[1] R. Bernabei et al., Proceed. of the 15th Bled Workshop "What Comes Beyond Standard Models" (December 2012), Vol. 13, No. 2, [arXiv:1301.6243v1[astro-ph.GA]]

[2] T. Piffi et al., Astronomy \& Astrophysics, Volume 562 (2014), [arXiv:1309.4293v3[astro-ph.GA]]

[3] J. Lavalle and S. Magni, accepted for publication in Phys. Rev. D, [arXiv:1411.1325[astro-ph.CO]]

[4] C.H. Faham et al. (LUX Collaboration), Proceedings for the 2014 Rencontres de Moriond (Cosmology Session), La Thuile, Italy, [arXiv:1405.5906[hep-ex]]

[5] R. Agnese et al. (SuperCDMS Collaboration), Phys.Rev.Lett. 112 (2014) 24, 241302

[6] G.Angloher et al., Eur.Phys.J. C74 (2014) 12, 3184, [arXiv:1407.3146v1[astro-ph.CO]]

[7] L. Hernquist, Astrophysical J., 356: 359-364, 1990 June 20

[8] M. Miyamoto and R. Nagai, Publ. Astron. Soc. Japan 27, 533-543 (1975)

[9] S. Gillessen et al., Astrophys.J. 692 (2009) 1075-1109, [arXiv:0810.4674[astro-ph]]

[10] R. Schönrich et al., Mon. Not R. Astron. Soc. 000,1-5 (2009), [arXiv:0912.3693v1[astro-ph.GA]]

[11] M. J. Reid et al., Astrophysical J., Volume 700, Issue 1 (2009), [arXiv:0902.3913[astro-ph.GA]]

[12] O. Bienaymé et al., Astronomy \& Astrophysics, Volume 571 (2014), [arXiv:1406.6896[astro-ph.GA]] 